\title{
Magnetic coupling in asymmetric FeCoV/Ru/FeNi trilayers
}

\author{
Yajun Wei, ${ }^{1}$ Somnath Jana, ${ }^{2}$ Rimantas Brucas, ${ }^{1}$ Yevgen Pogoryelov, ${ }^{2}$ Mojtaba Ranjbar, ${ }^{3}$ \\ Randy K. Dumas, ${ }^{3}$ Peter Warnicke, ${ }^{4}$ Johan Åkerman, ${ }^{3}$ Dario A. Arena, ${ }^{5}$ Olof Karis, ${ }^{2}$ \\ and Peter Svedlindh ${ }^{1, a)}$ \\ ${ }^{1}$ Department of Engineering Sciences, Uppsala University, 75121 Uppsala, Sweden \\ ${ }^{2}$ Department of Physics and Astronomy, Uppsala University, 75120 Uppsala, Sweden \\ ${ }^{3}$ Department of Physics, University of Gothenburg, 41296 Gothenburg, Sweden \\ ${ }^{4}$ Swiss Light Source, Paul Scherrer Institute, 5232 Villigen, Switzerland \\ ${ }^{5}$ National Synchrotron Light Source, Brookhaven National Laboratory, Upton, New York 11973, USA
}

(Presented 6 November 2013; received 23 September 2013; accepted 7 November 2013; published online 10 February 2014)

\begin{abstract}
We have investigated the magnetic anisotropy and interlayer coupling in trilayer films of permendur $(100 \AA) / \mathrm{Ru} /$ permalloy $(100 \AA)$, with the thickness of the Ru spacer varying from 0 to $200 \AA$. While the permendur/permalloy sample exhibits a small in-plane uniaxial magnetic anisotropy with $H_{u}=27 \mathrm{Oe}$, all trilayers are magnetically isotropic in-plane. Results from hysteresis loop and ferromagnetic resonance measurements were fitted to a micromagnetic model, with the results indicating that all the films are ferromagnetically coupled except the one with $10 \AA$ $\mathrm{Ru}$ spacer, which shows antiferromagnetic coupling. The trilayers with Ru spacer layer thickness larger than $20 \AA$ exhibit only very weak ferromagnetic coupling. () 2014 AIP Publishing LLC. [http://dx.doi.org/10.1063/1.4864743]
\end{abstract}

\section{INTRODUCTION}

A trilayer system with two ferromagnetic (FM) layers separated by a non-magnetic (NM) spacer layer is of great interest due to its use in applications such as magnetic recording devices and non-volatile magnetic random memories. $^{1,2}$ The interlayer coupling between the two FM layers plays an important role for engineering the magnetic properties of the trilayer systems and there have been numerous studies on coupled trilayers. However, most of the investigations have been carried on symmetrical trilayers with the two FM layers made from the same material. Reports on the exchange coupling in trilayers with different FM layers such as $\mathrm{Fe} / \mathrm{Cu} / \mathrm{Ni}^{3}$ and $\mathrm{Ni} / \mathrm{Cu} / \mathrm{Co}^{4}$ are more scarce. In this paper, we present our investigation of the interlayer coupling in trilayer films of permendur(Pmd)/Ru/permalloy(Py). The $\mathrm{Pmd} / \mathrm{Ru} / \mathrm{Py}$ system enables us to perform layer resolved spin dynamic studies using time-resolved X-ray Magnetic Circular Dichroism (XMCD) measurements, which will be reported later.

\section{EXPERIMENTAL}

The films were deposited at room temperature using dc magnetron sputtering. The base pressure of deposition was $5 \times 10^{-8}$ Torr. The thin films consist of $\mathrm{Si} / \operatorname{Pmd}(100 \AA) /$ $\mathrm{Ru}\left(t_{\mathrm{Ru}} \AA\right) / \mathrm{Py}(100 \AA)$, with a $10 \AA \mathrm{Ru}$ layer added as cap. The chemical composition of the Pmd and Py alloys is $\mathrm{Fe}_{49} \mathrm{Co}_{49} \mathrm{~V}_{2}$ and $\mathrm{Fe}_{19} \mathrm{Ni}_{81}$, respectively. The Ru layer thickness was varied between 0 and $200 \AA$. The Ru spacer layer was deposited at low sputtering rate $(0.4 \AA / \mathrm{s})$ and low Ar gas pressure ( $3 \mathrm{mTorr})$ for optimal uniformity and interface smoothness.

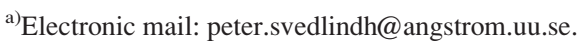

Room temperature magnetization versus field curves were measured using a SQUID magnetometer. We also performed XMCD studies at the Fe, Ni, and Co $L_{2,3}$ edges. This element specific technique allows us to determine the magnetic state of the separate magnetic layers as each of them contains two different magnetic elements; $\mathrm{Ni}$ in the Py layer and $\mathrm{Co}$ in the Pmd layer. The samples were also studied in a field-sweep cavity ferromagnetic resonance (FMR) system with an RF frequency of $9.8 \mathrm{GHz}$. Angle dependent FMR measurements were performed using a goniometer, keeping the DC magnetic field parallel to the film plane. FMR spectra using a vector network analyzer based FMR system (VNA-FMR) were additionally recorded. Frequency scans at different magnetic fields ranging from 0.2 to $1.2 \mathrm{kOe}$ were performed.

\section{MICROMAGNETIC MODEL}

We consider a trilayer system with two FM layers of the same thickness $t$, which are spaced by a NM Ru layer with thickness $t_{\mathrm{Ru}}$. The coordinate system and geometry deployed in the study are shown in the left top inset of Fig. 1. The external magnetic field $H$ is applied along the $x$-axis. Due to the demagnetizing field, which is much larger than any possible perpendicular anisotropy field, the magnetization in the two FM layers will be confined to the film plane. Taking into account the Zeeman energy, exchange energy, and demagnetizing energy, the total energy per unit area of the system is

$$
\begin{aligned}
\varepsilon_{\mathrm{tot}} & =\varepsilon_{\mathrm{zee}}+\varepsilon_{\mathrm{ex}}+\varepsilon_{\mathrm{dem}} \\
& =-t H\left(M_{A} \cos \phi_{A}+M_{B} \cos \phi_{B}\right)-J_{1} \cos \left(\phi_{A}-\phi_{B}\right) \\
& -J_{2} \cos ^{2}\left(\phi_{A}-\phi_{B}\right)-2 \pi t\left(M_{A}^{2} \cos ^{2} \theta_{A}+M_{B}^{2} \cos ^{2} \theta_{B}\right),
\end{aligned}
$$

where $J_{1}$ and $J_{2}$ are the bilinear and biquadratic coupling constants. When $J_{1}$ dominates over $J_{2}$, a positive (negative) $J_{1}$ corresponds to ferromagnetic (antiferromagnetic) coupling. 


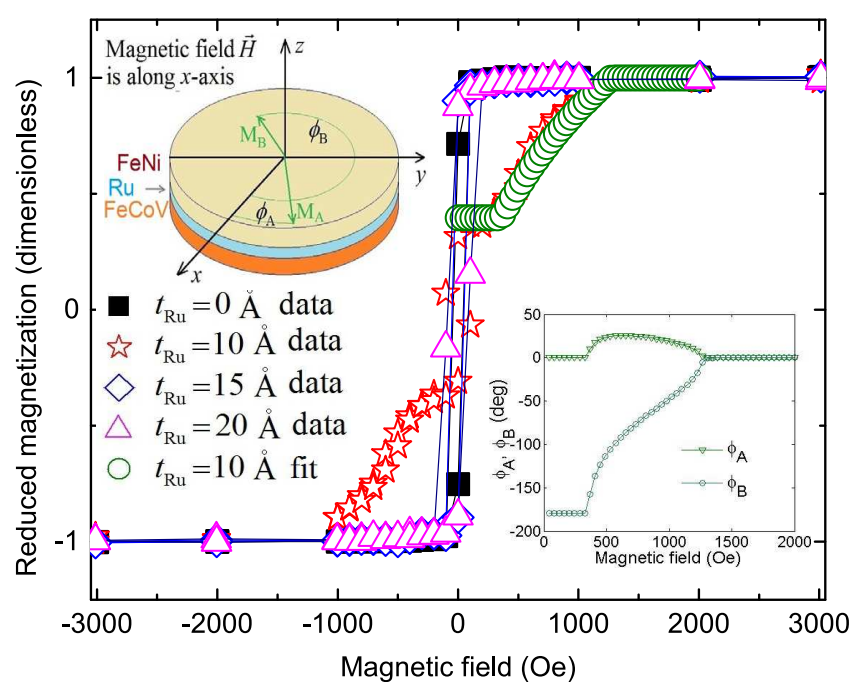

FIG. 1. Some typical magnetization curves of the $\mathrm{Pmd} / \mathrm{Ru} / \mathrm{Py}$ trilayers. The top left inset shows the geometry and coordinate system of the film samples. The right bottom inset shows $\phi_{A}$ and $\phi_{B}$ as a function of external field for the $t_{\mathrm{Ru}}=10 \AA$ sample.

For a given set of parameters describing the trilayer system $\left(J_{1}, J_{2}, M_{A}\right.$, and $\left.M_{B}\right)$, the equilibrium orientation of the magnetizations $\left(\phi_{A}, \phi_{B}\right)$ at any external field can be calculated numerically by finding the values of $\phi_{A}$ and $\phi_{B}$ minimizing $\varepsilon_{\text {tot }}$. Using these values, the static magnetization of the film is given by

$$
m=\frac{M(H)}{M_{s}}=\frac{M_{A} \cos \phi_{A}+M_{B} \cos \phi_{B}}{M_{A}+M_{B}} .
$$

In the FMR experiments, a microwave signal generating an RF magnetic field $\vec{h}$ that is perpendicular to the static field is used. Resonance absorption occurs when the microwave frequency matches the natural frequency of the system, which is determined by the magnetic properties of the sample and the static magnetic field. By substituting the energy density expression $\varepsilon_{\text {tot }}$ and the equilibrium angles into the Suhl Smit equation $^{5}$ and following a similar analysis as in Ref. 6 , the resonance condition is obtained as a fourth order polynomial equation of the microwave angular frequency $\omega$, which reads

$$
a \omega^{4}+c \omega^{2}+e=0 .
$$

The coefficients of the 4th order polynomial equation are

$$
\begin{aligned}
a= & \left(\frac{t^{2} M_{A} M_{B}}{\gamma_{A} \gamma_{B}}\right)^{2}, \\
c= & -t^{2} M_{A} M_{B}\left[\left(\frac{h_{1}^{A} h_{2}^{A}}{\gamma_{B}^{2}}+\frac{h_{1}^{B} h_{2}^{B}}{\gamma_{A}^{2}}\right)+\frac{2 C_{0} C_{2}}{\gamma_{A} \gamma_{B}}\right. \\
& +C_{1}\left(\frac{t M_{A} h_{2}^{B}}{\gamma_{A}^{2}}+\frac{t M_{B} h_{2}^{A}}{\gamma_{B}^{2}}\right)+C_{2}\left(\frac{t M_{A} h_{1}^{B}}{\gamma_{A}^{2}}+\frac{t M_{B} h_{1}^{A}}{\gamma_{B}^{2}}\right) \\
& \left.+C_{1} C_{2}\left(\frac{M_{A}}{M_{B} \gamma_{A}^{2}}+\frac{M_{B}}{M_{A} \gamma_{B}^{2}}\right)\right], \\
e= & {\left[t^{2} M_{A} M_{B}+C_{2}\left(t M_{A} h_{2}^{A}+t M_{B} h_{2}^{B}\right)\right] } \\
& \times\left[t^{2} M_{A} M_{B} h_{1}^{A} h_{1}^{B}+C_{1}\left(t M_{A} h_{1}^{A}+t M_{B} h_{1}^{B}\right)+\left(C_{1}^{2}-C_{0}^{2}\right)\right],
\end{aligned}
$$

with $\gamma_{A}$ and $\gamma_{B}$ being the gyromagnetic ratio of the FM layer $\mathrm{A}$ and $\mathrm{B}$, respectively. The parameters in Eq. (4) are defined as $C_{0}=J_{1}+2 J_{2} \cos \left(\phi_{A}-\phi_{B}\right), C_{1}=J_{1} \cos \left(\phi_{A}-\phi_{B}\right)+2 J_{2} \cos ^{2}$ $\left(\phi_{A}-\phi_{B}\right), \quad C_{2}=J_{1} \cos \left(\phi_{A}-\phi_{B}\right)+2 J_{2} \cos 2\left(\phi_{A}-\phi_{B}\right), h_{1}^{A(B)}$ $=H \cos \phi_{A(B)}+4 \pi M_{A(B)}$, and $h_{2}^{A(B)}=H \cos \phi_{A(B)}$.

Among the four solutions of Eq. (3), only two are physically meaningful. These two resonances are referred to as the acoustic mode, which corresponds to the two magnetization vectors in the two FM layers resonating in-phase and the optic mode, which corresponds to the two magnetizations in the two FM layers resonating out-of-phase.

\section{RESULTS AND DISCUSSION}

The results from angle dependent field-sweep cavityFMR measurements show that all trilayer samples are isotropic in-plane. The Pmd/Py bilayer sample exhibits a small in-plane uniaxial anisotropy characterized by an anisotropy field of $H_{u}=27 \mathrm{Oe}$. The in-plane magnetization curves for some of the films are presented in Fig. 1. The hysteresis loops of the films indicate that all films are ferromagnetically coupled except the one with $10 \AA \mathrm{Ru}$ spacer, which shows antiferromagnetic coupling instead. In addition, the XMCD studies performed in remanence were found to agree with the hysteresis measurements. Analyzing the difference spectra between the left and right circularly polarized light collected at the $\mathrm{Ni}$ and $\mathrm{Co} L$ edges (not shown), we find that $\mathrm{Ni}$ and Co moments are aligned antiparallel to each other for the sample with $10 \AA$ thick Ru spacer, while for the other samples they are aligned in parallel. The antiferromagnetically coupled sample is characterized by a bilinear coupling constant of $-0.57 \mathrm{erg} / \mathrm{cm}^{2}$ and a small biquadratic coupling constant of $-0.05 \mathrm{erg} / \mathrm{cm}^{2}$. The coupling constants were obtained by matching the calculated magnetization curve, using Eqs. (1) and (2), with the experimental data; the right bottom inset in Fig. 1 shows $\phi_{A}$ and $\phi_{B}$ as a function of external field for this sample. The saturation magnetization values used in the calculations are $4 \pi M_{A}=22 \mathrm{kG}$ for $\mathrm{Pmd}^{7}$ and $4 \pi M_{B}=9.5 \mathrm{kG}$ for Py. ${ }^{8}$

Figure 2 shows the dispersion relations (resonance frequency versus magnetic field) for some of the trilayer samples. The coupling constants were obtained by fitting the experimental cavity-FMR and VNA-FMR results to Eq. (3); the bilinear coupling constants are presented in Fig. 3. The simulated resonance frequency versus magnetic field curves fit the experimental data quite well, without introduction of the biquadratic coupling constant for any of the ferromagnetically coupled samples. However, for the antiferromagnetically coupled sample, a small biquadratic coupling constant of $-0.05 \mathrm{erg} / \mathrm{cm}^{2}$ was necessary to fit the experimental data. It is important to note that this value is much smaller compared to the bilinear coupling constant, implying that the coupling is dominated by the bilinear contribution. Interestingly, the best fit obtained from the FMR data yields $J_{1}=-0.62 \mathrm{erg} / \mathrm{cm}^{2}$, slightly different from the one obtained from the magnetization curves. This can be explained by the fact that in the micromagnetic model, discussed in Sec. III, we treated each layer of the film as a single magnetic domain 


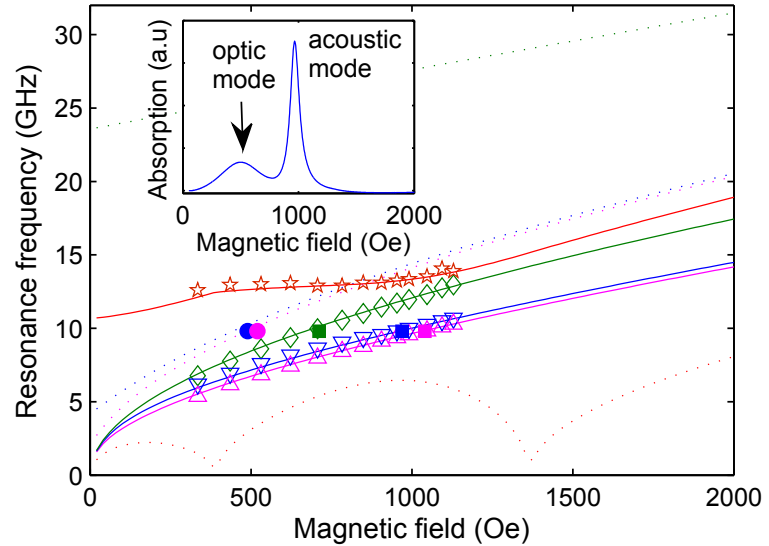

FIG. 2. The dispersion relations of $\mathrm{Pmd} / \mathrm{Ru} / \mathrm{Py}$ trilayers with $t_{\mathrm{Ru}}=0 \AA$ (green), $t_{\mathrm{Ru}}=10 \AA$ (red), $t_{\mathrm{Ru}}=15 \AA$ (blue), and $t_{\mathrm{Ru}}=20 \AA$ (magenta). Open and solid markers are experimental data obtained from VNA-FMR and cavity-FMR, respectively. Lines correspond to simulated curves using the micromagnetic model discussed in Sec. III, with solid lines representing the acoustic modes and dotted lines the optic modes. The inset shows the cavity FMR spectrum of the sample with $t_{\mathrm{Ru}}=15 \AA$.

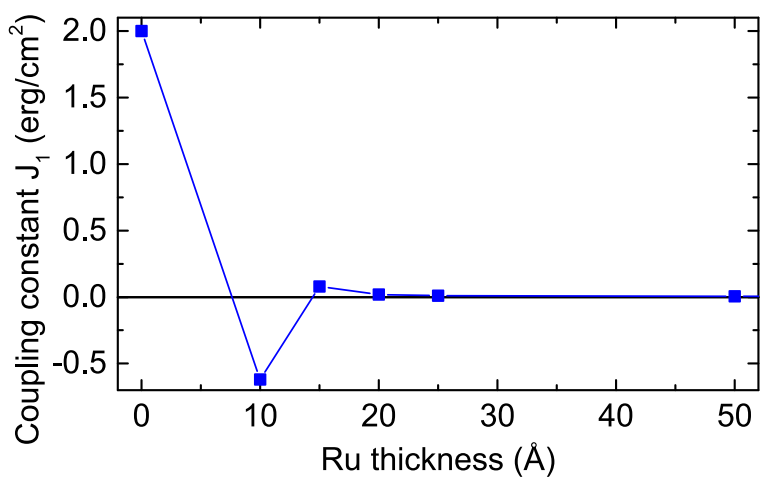

FIG. 3. Bilinear coupling constants as a function of $t_{\mathrm{Ru}}$. The solid line is a guide to the eye.

and neglected effects of a multidomain state. Another comment we would like to add is that the VNA-FMR setup was unable to resolve the optic mode resonance peaks due to limitations in instrument sensitivity and the low intensity of the optic mode resonance..$^{9,10}$

As one can see from Fig. 3, the FM coupling for the Pmd/Py sample is comparably strong with $J_{1}=2 \mathrm{erg} / \mathrm{cm}^{2}$. The coupling constant changes sign for the sample with $10 \AA$ $\mathrm{Ru}$ thickness but changes back to FM coupling for larger Ru thickness. For Ru spacer thickness larger than $20 \AA$, the static Ruderman-Kittel-Kasuya-Yosida (RKKY) coupling strength vanishes. Despite this, the films with $\mathrm{Ru}$ spacer thickness in the range of $20 \AA-50 \AA$ are still coupled as the FMR spectra do not reveal two distinct resonance peaks for the Pmd and Py layers. This is tentatively explained by so-called dynamic exchange coupling interaction. ${ }^{11}$ Further studies are under way. The coupling constants of our asymmetric trilayers exhibit values similar to that of symmetric $\mathrm{Py} / \mathrm{Ru} / \mathrm{Py}$ trilayers but its dependence on the $\mathrm{Ru}$ thickness is shifted toward to the thicker end. ${ }^{12,13}$

\section{CONCLUSION}

In conclusion, we have investigated the magnetic anisotropy and interlayer coupling in $\mathrm{Pmd} / \mathrm{Ru} / \mathrm{Py}$ trilayers grown on silicon substrates. The film without $\mathrm{Ru}$ spacer shows a weak in-plane uniaxial anisotropy with an anisotropy field of $27 \mathrm{Oe}$, while all of the trilayer samples were magnetically isotropic in-plane. The films are all ferromagnetically coupled except the one with $10 \AA \mathrm{Ru}$ spacer, which shows a relatively strong antiferromagnetic coupling characterized by a bilinear coupling constant $J_{1}=-0.6 \mathrm{erg} / \mathrm{cm}^{2}$ and biquadratic coupling constant $J_{2}=-0.05 \mathrm{erg} / \mathrm{cm}^{2}$. The bilayer $\mathrm{Pmd} / \mathrm{Py}$ sample is strongly ferromagnetically coupled with a coupling constant of about $2 \mathrm{erg} / \mathrm{cm}^{2}$. The static RKKY coupling strength vanishes for $\mathrm{Ru}$ spacer thickness larger than $20 \AA$.

\section{ACKNOWLEDGMENTS}

This work was supported by the Swedish Research Council, the Knut and Alice Wallenberg Foundation, and the Swedish Foundation for Strategic Research.

${ }^{1}$ S. Wolf, D. Awschalom, R. Buhrman, J. Daughton, S. Von Molnar, M. Roukes, A. Y. Chtchelkanova, and D. Treger, Science 294, 1488 (2001).

${ }^{2}$ B. Engel, J. Akerman, B. Butcher, R. Dave, M. DeHerrera, M. Durlam, G. Grynkewich, J. Janesky, S. Pietambaram, N. Rizzo et al., IEEE Trans. Magn. 41, 132 (2005).

${ }^{3}$ W. Weber, R. Allenspach, and A. Bischof, Europhys. Lett. 31, 491 (1995).

${ }^{4}$ J. Lindner, Z. Kollonitsch, E. Kosubek, M. Farle, and K. Baberschke, Phys. Rev. B 63, 094413 (2001).

${ }^{5}$ H. Suhl, Phys. Rev. 97, 555 (1955).

${ }^{6}$ A. Layadi, Phys. Rev. B 65, 104422 (2002).

${ }^{7}$ Y. Wei, R. Brucas, K. Gunnarsson, I. Harward, Z. Celiniski, and P. Svedlindh, J. Phys. D: Appl. Phys. 46, 495002 (2013).

${ }^{8}$ E. Klokholm and J. Aboaf, J. Appl. Phys. 52, 2474 (1981).

${ }^{9}$ J. Cochran, J. Rudd, W. Muir, B. Heinrich, and Z. Celinski, Phys. Rev. B 42, 508 (1990).

${ }^{10}$ S. Rezende, C. Chesman, M. Lucena, A. Azevedo, F. De Aguiar, and S. Parkin, J. Appl. Phys. 84, 958 (1998).

${ }^{11}$ B. Heinrich, Y. Tserkovnyak, G. Woltersdorf, A. Brataas, R. Urban, and G. E. Bauer, Phys. Rev. Lett. 90, 187601 (2003).

${ }^{12}$ J. Fassbender, F. Nörtemann, R. Stamps, R. Camley, B. Hillebrands, G. Güntherodt, and S. Parkin, Phys. Rev. B 46, 5810 (1992).

${ }^{13}$ M. Belmeguenai, T. Martin, G. Woltersdorf, M. Maier, and G. Bayreuther, Phys. Rev. B 76, 104414 (2007). 